\title{
DEKOMPOSISI SUPER AJAIB BERBENTUK LINTASAN DARI AMALGAMASI GRAF SIKLUS
}

\section{(A PATH-SUPER MAGIC DECOMPOSITION OF THE VERTEX AMALGAMATION OF SOME CYCLES)}

\author{
Sigit Pancahayani ${ }^{1}$ \\ ${ }^{1}$ Institut Teknologi Kalimantan, spancahayani@itk.ac.id
}

\begin{abstract}
Abstrak
Misalkan $G=\operatorname{Amal}\left\{C_{n}\right\}_{t}$ adalah graf sederhana, berhingga, dan terhubung yang diperoleh dengan melekatkan $t$ buah graf siklus berukuran $n$ pada sebuah titik tetap $v_{0}$ sebagai terminalnya. Jika $H$ adalah subgraf dari $G$, maka $G$ dikatakan memuat dekomposisi $H$ super ajaib jika terdapat koleksi subgraf dari $G$, yaitu $\mathcal{H}=\left\{H_{1}, H_{2}, \cdots\right.$ ,$\left.H_{n}\right\}$ yang memenuhi untuk setiap $i, j \in \mathbb{N}$ berlaku $E\left(H_{i}\right) \cap E\left(H_{j}\right)=$ $\varnothing$ jika $i \neq j, \quad \cup_{i} H_{i}=G$, dan $\forall_{i} H_{i} \cong H$, fungsi bijeksi $f: V(G) \cup$ $E(G) \rightarrow 1,2,3, \cdots,|V(G)|+|E(G)|, \quad$ dan $\quad f(V(G))=\{1,2,3, \cdots$ , $|V(G)|\}$ sehingga setiap subgraf $H_{i} \in H$ memiliki bobot yang sama sebesar $k$, sebagai konstanta ajaib. Pada penelitian ini, digunakan multiset dengan konsep keseimbangan- $k$ untuk menunjukkan bahwa $\operatorname{Amal}\left\{C_{n}\right\}_{t}$ memuat dekomposisi $P_{n+1}$ super ajaib.
\end{abstract}

Kata kunci: Amalgamasi; Dekomposisi; Graf Siklus; Lintasan

\begin{abstract}
Let $G=$ Amal $\left\{C_{n}\right\}_{t}$ be a simple, finite, and connected graph which is constructed by joining $t$ copy of cycles of order $n$ onto a fixed point, $v_{0}$, called terminal vertex. Let $H$ be a subgraph of $G$. $G$ admits a super magic $H$ decomposition if there exists $\mathcal{H}=\left\{H_{1}, H_{2}, \cdots, H_{n}\right\}$ a collection of subgraphs of $G$ which satisfies $E\left(H_{i}\right) \cap E\left(H_{j}\right)=\emptyset$ for $i \neq j$ where $i, j \in \mathbb{N}, \cup_{i} H_{i}=G$, and $\forall_{i} H_{i} \cong H$, a bijection mapping $f: V(G) \cup E(G) \rightarrow 1,2,3, \cdots,|V(G)|+|E(G)|, \quad$ and $\quad f(V(G))=$ $\{1,2,3, \cdots,|V(G)|\}$ such that every subgraph $H_{i} \in H$ has the same weight of valuation $k$ as a magic constant. This research uses multiset concept of $k$-balance to show that Amal $\left\{C_{n}\right\}_{t}$ admits a $P_{n+1}-$ super magic decomposition.
\end{abstract}

Keywords: Amalgamation; Decomposition; Cycles; Path

\section{PENDAHULUAN}

Penelitian ini difokuskan pada graf terhubung yang sederhana dan berhingga. Misalkan $G=(V, G)$ adalah suatu graf dan $H$ adalah subgraf dari $G$. Graf $G$ disebut memuat dekomposisi $H$ jika terdapat suatu koleksi subgraf dari $G$, $\mathcal{H}=\left\{H_{1}, H_{2}, \cdots, H_{n}\right\}$ yang memenuhi syarat jika $i, j \in \mathbb{N}$ dengan $i \neq j$, maka berlaku $E\left(H_{i}\right) \cap E\left(H_{j}\right)=\emptyset, \cup_{i} H_{i}=G$, dan $\forall_{i} H_{i} \cong H$. Lebih jauh, $G$ memuat dekomposisi $H$ ajaib jika terdapat fungsi bijektif $f: V(G) \cup E(G) \rightarrow 1,2,3, \cdots$ , $|V(G)|+|E(G)|$ sedemikian sehingga setiap subgraf $H_{i} \in \mathcal{H}$ memiliki bobot yang sama, yaitu $w\left(H_{i}\right)=\sum_{v \in V\left(H_{i}\right)} f(v)+\sum_{e \in E\left(H_{i}\right)} f(e)=k$, dengan $k$ adalah 
konstanta ajaib. Selanjutnya, $G$ memuat dekomposisi $H$ super ajaib jika $f(V(G))=\{1,2,3, \cdots,|V(G)|\}$. Beberapa hasil mengenai dekomposisi $H$ ajaib diberikan oleh (Salman \& Maryati, 2010), (Maryati, 2011), (Inayah, Lladó, \& Moragas, 2012), (Maryati, Salman, \& Baskoro, 2013), dan (Roswitha, Baskoro, Maryati, Kurdhi, \& Susanti, 2013).

\section{METODE}

Maryati (2011) juga memperkenalkan suatu konsep keseimbangan- $k$ pada sebuah himpunan. Misalkan $X$ adalah himpunan. Didefinisikan $\sum X=\sum_{x \in X} x$ sebagai sumset, yaitu jumlahan dari seluruh anggota dari himpunan $X$. Didefinisikan pula $\mathcal{M}$ sebagai multiset, yaitu himpunan yang memungkinkan untuk memiliki anggota yang sama. Misalkan $m, n$, dan $k_{i}$ adalah bilangan bulat dengan $i \in[1, n], \mathcal{M}=\left\{m_{1}^{k_{1}}, m_{2}^{k_{2}}, \ldots, m_{n}^{k_{n}}\right\}$ adalah multiset yang memiliki anggota $m_{i}$ sebanyak $k_{i}$. Pada dua buah multiset $X_{i}=\left\{x_{1}^{k_{i}}, x_{2}^{k_{i}}, \ldots, x_{n}^{k_{i}}\right\}$, dengan $i \in[1, n]$ dan $Y_{j}=\left\{y_{1}^{k_{j}}, y_{2}^{k_{j}}, \ldots, y_{m}^{k_{j}}\right\}$, dengan $j \in[1, m]$, didefinisikan $X_{i} \biguplus Y_{j}=$ $\left\{x_{1}^{k_{i}}, x_{2}^{k_{i}}, \ldots, x_{n}^{k_{i}}, y_{1}^{k_{j}}, y_{2}^{k_{j}}, \ldots, y_{m}^{k_{j}}\right\}$. Jika $k \in \mathbb{N}$, multiset $\mathcal{M}$ memenuhi keseimbangan- $k$ jika terdapat $k$ buah $M_{i}$ sebagai subhimpunan $\mathcal{M}$ sedemikian sehingga $\left|M_{i}\right|=\frac{|\mathcal{M}|}{k}, \sum M_{i}=\frac{\sum \mathcal{M}}{k}$, dan $\biguplus_{i=1}^{k} M_{i}=\mathcal{M}$.

\section{HASIL DAN PEMBAHASAN}

Misalkan $\mathcal{C}=\left\{C_{n, i} \mid i=1,2,3, \ldots, t\right\}$ adalah koleksi graf siklus dan setiap sikus memiliki sebuah titik tetap $v_{0}$ yang disebut terminal. Amalgamasi titik dari sejumlah siklus di $\mathcal{C}$, dinotasikan sebagai $\operatorname{Amal}\left\{C_{n, i}\right\}_{i=1}^{t}$, adalah graf yang diperoleh dengan cara melekatkan titik-titik terminal dari setiap siklus. Jika setiap $C_{n, i}$ isomorfis terhadap $C_{n}$, maka amalgamasi titik dari $t$ buah $C_{n}$ secara sederhana ditulis $\operatorname{Amal}\left\{C_{n}\right\}_{t}$.

Dalam penelitian ini, diperlukan format pelabelan titik dan sisi dalam $\operatorname{Amal}\left\{C_{n}\right\}_{t}$ sebagai berikut:

- Untuk $i=1,2,3, \ldots, t$, setiap siklus $C_{n, i}$ di $\operatorname{Amal}\left\{C_{n}\right\}_{t}$ disebut daun dengan label titik $V\left(C_{n, i}\right)=\left\{v_{0}, v_{1}^{i}, v_{2}^{i}, \ldots, v_{n-1}^{i}\right\} \quad$ dan $\quad E\left(C_{n, i}\right)=\left\{e_{j}^{i}=\right.$ $\left.v_{j-1}^{i} v_{j}^{i}, e_{n}^{i}=v_{n-1}^{i} v_{0} \mid j=1,2, \ldots, n-1\right\}$.

- Untuk $i=1,2,3, \ldots, t-1$, label untuk titik-titik di subgraf $P_{n+1}^{i}$ dari $\operatorname{Amal}\left\{C_{n}\right\}_{t}$ adalah $V\left(P_{n+1}^{i}\right)=\left\{v_{1}^{i}, v_{2}^{i}, \ldots, v_{n-1}^{i}, v_{0}, v_{1}^{i+1}\right\}$ dan $V\left(P_{n+1}^{t}\right)=$ $\left\{v_{1}^{t}, v_{2}^{t}, \ldots, v_{n-1}^{t}, v_{0}, v_{1}^{1}\right\}$

Dari format pelabelan titik dan sisi pada $\operatorname{Amal}\left\{C_{n}\right\}_{t}$ tersebut, terlihat bahwa untuk $i=1,2,3, \ldots, t, v_{0}$ muncul pada $t$ buah $P_{n+1}^{i}$ dan $v_{1}^{i}$ terdaftar sebanyak dua kali dari seluruh $P_{n+1}^{i}$.

\section{Teorema 1}

Misalkan $n \geq 3$ dan $t \geq 2$ adalah dua buah bilangan bulat positif. Maka $\operatorname{Amal}\left\{C_{n}\right\}_{t}$ memuat dekomposisi $P_{n+1}$ super ajaib dengan $k=n t(2 n-1)+$ $(2 n+1)$. 


\section{Bukti}

Perhatikan bahwa $\left|V\left(\operatorname{Amal}\left\{C_{n}\right\}_{t}\right)\right|=(n-1) t+1$ dan $\left|E\left(\operatorname{Amal}\left\{C_{n}\right\}_{t}\right)\right|=n t$. Kemudian, didefinisikan sebuah multiset

$$
\begin{gathered}
\mathcal{M}=\{1,1,2,2,3,3, \ldots, t, t, t+1, t+2, t+3, \ldots, \underbrace{(n-1) t+1, \ldots,(n-1) t+1}_{t \text { kali }}, \\
(n-1) t+2,(n-1) t+3, \ldots,(2 n-1) t+1\} .
\end{gathered}
$$

Untuk $i=1,2, \ldots, t-1$, misalkan $M_{i}$ dan $M_{t}$ adalah submultiset dari $\mathcal{M}$ sedemikian hingga $\left(\cup_{i} M_{i}\right) \biguplus M_{t}=\mathcal{M}$. Misalkan $M_{i}=\biguplus_{k=1}^{5} A_{k}^{i}$ dan $M_{t}=\biguplus_{k=1}^{5} A_{k}^{t}$ dengan

$$
\begin{aligned}
& A_{1}^{i}=\{j t+i \mid j=0,1,2,3, \ldots, n-2\} ; \\
& A_{2}^{i}=\{(n-1) t+1\} ; \\
& A_{3}^{i}=\{1+i\} ; \\
& A_{4}^{i}=\{(n+j) t+2-i \mid j=0,1,2,3, \ldots, n-2\} ; \\
& A_{5}^{i}=\{(2 n-1) t+1-i\} ; \\
& A_{1}^{t}=\{j t \mid j=1,2,3, \ldots, n-1\} ; \\
& A_{2}^{t}=\{(n-1) t+1\} ; \\
& A_{3}^{t}=\{1\} ; \\
& A_{4}^{t}=\{(n+j) t+2 \mid j=-1,0,1,2,3, \ldots, n-3\} ; \\
& \text { dan } \\
& A_{5}^{t}=\{(2 n-1) t+1\} ;
\end{aligned}
$$

Akibatnya, diperoleh

$$
\begin{aligned}
\sum M_{i}= & \sum A_{1}^{i}+\sum A_{2}^{i}+\sum A_{3}^{i}+\sum A_{4}^{i}+\sum A_{5}^{i} \\
= & \sum_{j=0}^{n-2}(j t+i)+(n-1) t+1+(1+i)+ \\
& \sum_{j=0}^{n-2}[(n+j) t+2-i]+[(2 n-1) t+1-i] \\
= & n t(2 n-1)+(2 n+1)
\end{aligned}
$$

dan

$$
\begin{aligned}
\sum M_{t} & =\sum A_{1}^{t}+\sum A_{2}^{t}+\sum A_{3}^{t}+\sum A_{4}^{t}+\sum A_{5}^{t} \\
& =\sum_{j=1}^{n-2} j t+[(n-1) t+1]+1+\sum_{j=-1}^{n-3}[(n+j) t+2]+[(2 n-1) t+1] \\
& =n t(2 n-1)+(2 n+1)
\end{aligned}
$$

Selanjutnya, untuk $i=1,2, \ldots, t$ dan $j=1,2, \ldots, n-1$, didefinisikan fungsi pelabelan pada titik sebagai berikut:

$f_{1}: V\left(\operatorname{Amal}\left\{C_{n}\right\}_{t}\right) \rightarrow\{1,2,3, \ldots, t,(n-1) t+1\}$

$v_{j}^{i} \mapsto(j-1) t+i$

$v_{0} \mapsto(n-1) t+1$

dan

$f_{2}: E\left(\operatorname{Amal}\left\{C_{n}\right\}_{t}\right) \rightarrow\{(n-1) t+2,(n-1) t+3, \ldots,(2 n-1) t+1\}$

$e_{j}^{i} \mapsto(n+j-2) t+2-i$ untuk $i=1,2, \ldots, t$ dan $j=2,3, \ldots, n$

$e_{1}^{i+1} \mapsto(2 n-1) t+1-i$

$e_{1}^{1} \mapsto(2 n-1) t+1$

Dengan demikian, himpunan label titik pada subgraf $P_{n+1}^{i} \subseteq \operatorname{Amal}\left\{C_{n}\right\}_{t}$ untuk $i=1,2, \ldots, t$ adalah $f_{1}\left(V\left(P_{n+1}^{i}\right)\right) \cup f_{2}\left(E\left(P_{n+1}^{i}\right)\right)=M_{i}$ dan $f_{1}\left(V\left(P_{n+1}^{t}\right)\right) \cup$ $f_{2}\left(E\left(P_{n+1}^{t}\right)\right)=M_{t}$. Oleh karena itu, $\operatorname{Amal}\left\{C_{n}\right\}_{t}$ memuat dekomposisi $P_{n+1}$ super ajaib dengan konstanta ajaib $k=n t(2 n-1)+(2 n+1)$. 


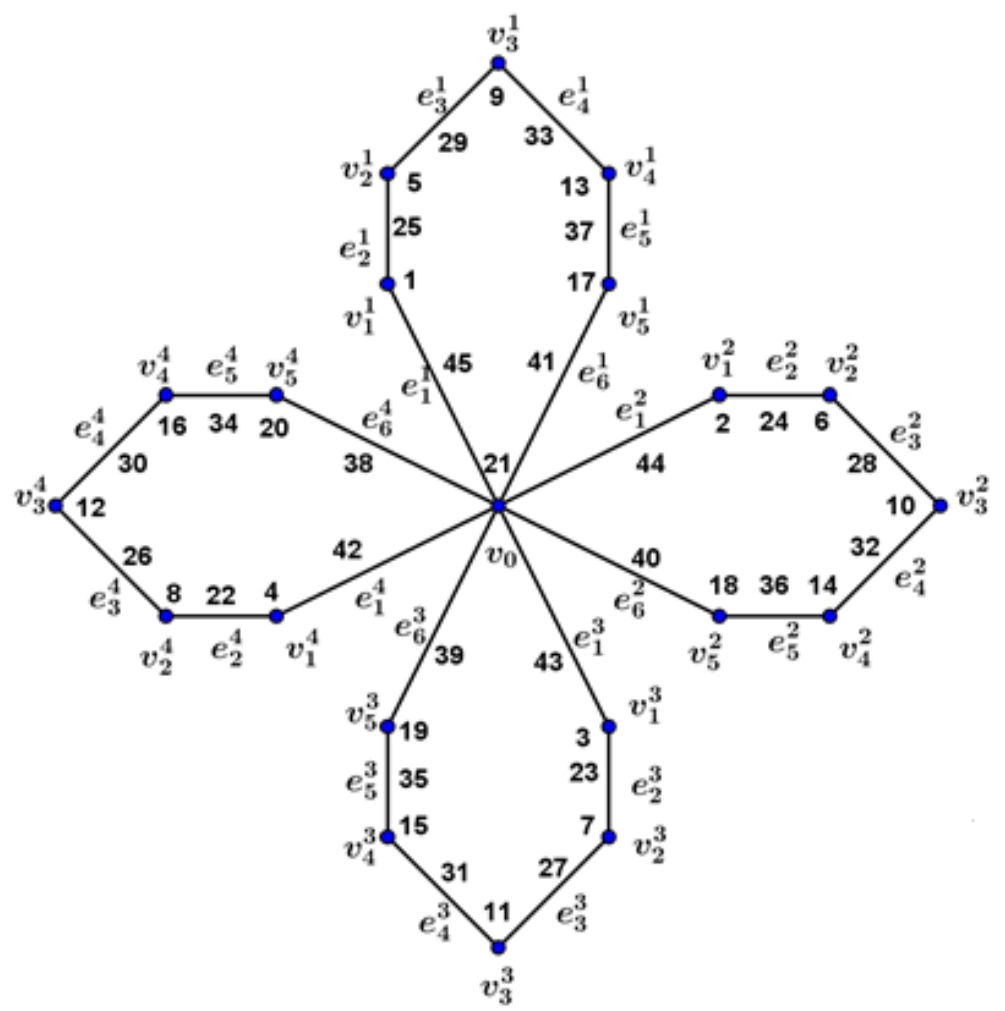

Gambar 1. Pelabelan Titik dan Sisi pada Graf $\left.\operatorname{Amal}_{\left\{C_{6}\right.}\right\}_{4}$

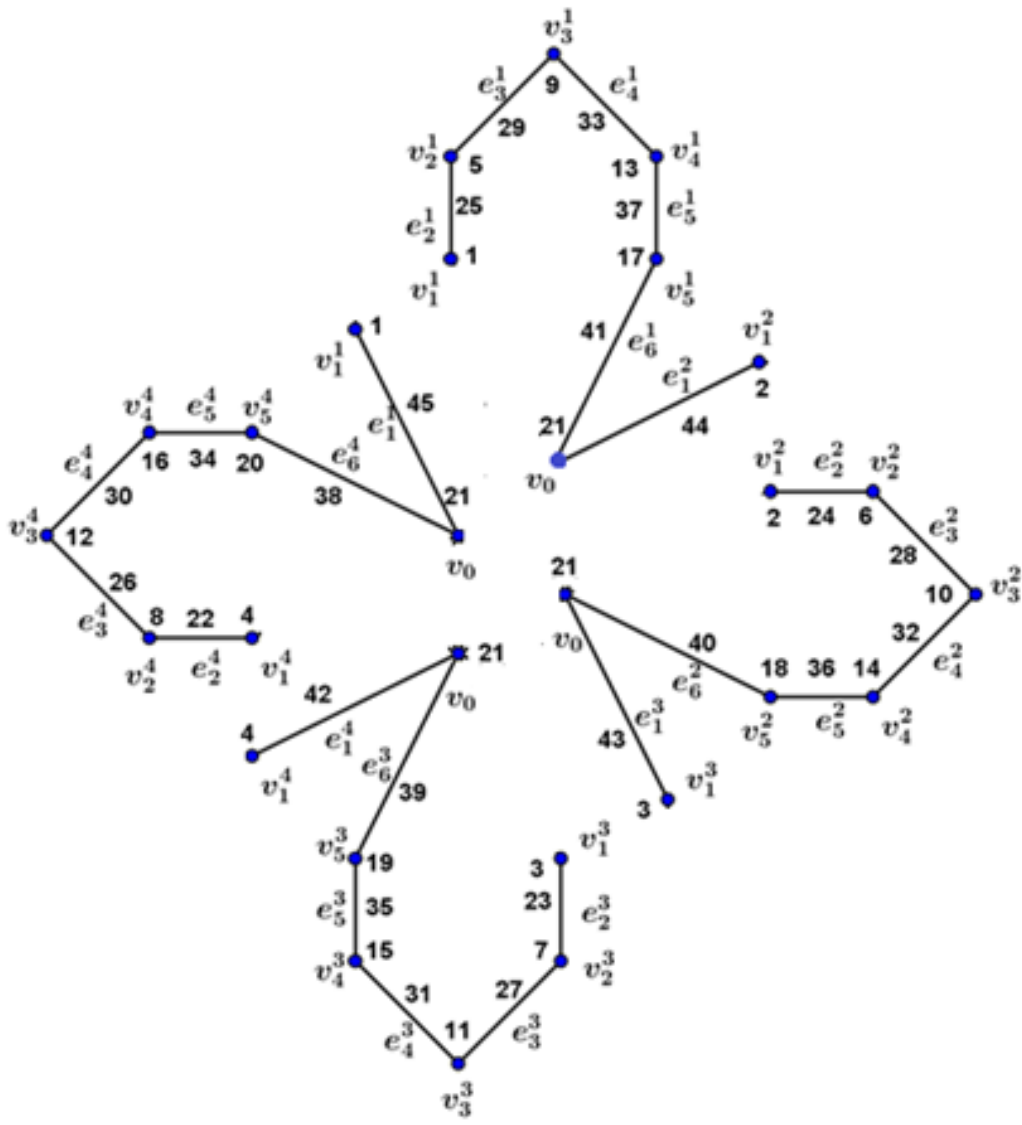

Gambar 2. Dekomposisi Super Ajaib pada Graf $\mathrm{Amal}\left\{\mathrm{C}_{6}\right\}_{4}$ dengan Konstanta Ajaib $k=277$ 


\section{Teorema 2}

Misalkan $n \geq 3$ dan $t \geq 2$ adalah dua buah bilangan bulat positif. Maka $\operatorname{Amal}\left\{C_{n}\right\}_{t}$ memuat dekomposisi $P_{n+1}$ super ajaib dengan $k=2 n t(n-1)+$ $(3 n+t+1)$.

\section{Bukti}

Definisikan sebuah multiset

$$
\begin{gathered}
\mathcal{N}=\{\underbrace{1, \ldots, 1}_{t \text { kali }}, 2,2,3,4, \ldots, t, t, t+1, t+1, t+2, t+3, \ldots,(n-1) t+1,(n-1) t \\
+2,(n-1) t+3, \ldots,(2 n-1) t+1\}
\end{gathered}
$$

Untuk $i=1,2, \ldots, t-1$, misalkan $N_{i}$ dan $N_{t}$ adalah submultiset dari $\mathcal{N}$ sedemikian hingga $\left(\bigcup_{i} N_{i}\right) \biguplus N_{t}=\mathcal{N}$. Misalkan $N_{i}=\biguplus_{k=1}^{5} B_{k}^{i}$ dan $N_{t}=\biguplus_{k=1}^{5} B_{k}^{t}$ dengan

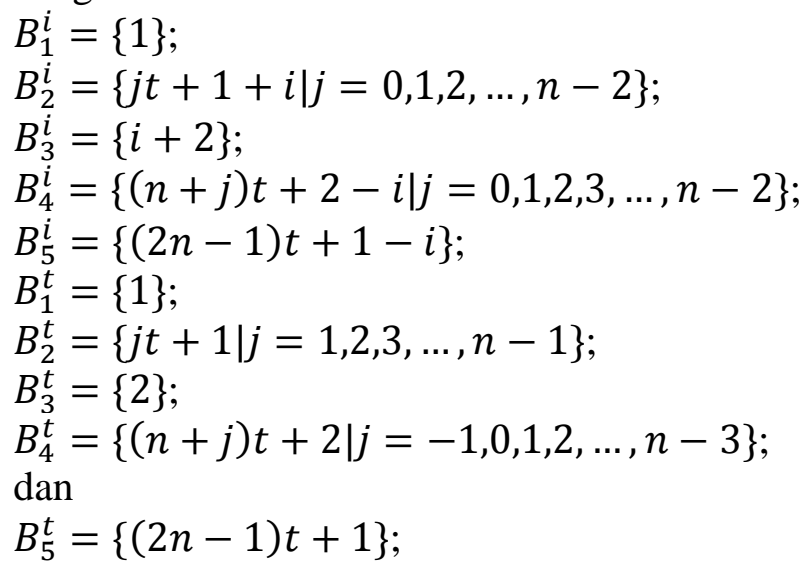

Akibatnya, diperoleh

$$
\begin{aligned}
\sum N_{i}= & \sum B_{1}^{i}+\sum B_{2}^{i}+\sum B_{3}^{i}+\sum B_{4}^{i}+\sum B_{5}^{i} \\
= & 1+\sum_{j=0}^{n-2}(j t+1+i)+(i+2)+ \\
& \sum_{j=0}^{n-2}[(n+j) t+2-i]+[(2 n-1) t+1-i] \\
= & 2 n t(n-1)+(3 n+t+1) \\
\text { dan } & \\
\sum N_{t}= & \sum B_{1}^{t}+\sum B_{2}^{t}+\sum B_{3}^{t}+\sum B_{4}^{t}+\sum B_{5}^{t} \\
= & 1+\sum_{j=1}^{n-1}(j t+1)+2+\sum_{j=-1}^{n-3}[(n+j) t+2]+[(2 n-1) t+1] \\
= & 2 n t(n-1)+(3 n+t+1)
\end{aligned}
$$

Selanjutnya, untuk $i=1,2, \ldots, t$ dan $j=1,2, \ldots, n-1$, didefinisikan fungsi pelabelan pada titik sebagai berikut:

$$
\begin{aligned}
& g_{1}: V\left(\operatorname{Amal}\left\{C_{n}\right\}_{t}\right) \rightarrow\{1,2,3, \ldots, t,(n-1) t+1\} \\
& v_{j}^{i} \mapsto(j-1) t+i+1 \\
& v_{0} \mapsto 1 \\
& \text { dan } \\
& g_{2}: E\left(\operatorname{Amal}\left\{C_{n}\right\}_{t}\right) \rightarrow\{(n-1) t+2,(n-1) t+3, \ldots,(2 n-1) t+1\} \\
& e_{j}^{i} \mapsto(n+j-2) t+2-i \text { untuk } i=1,2, \ldots, t \text { dan } j=2,3, \ldots, n \\
& e_{1}^{i+1} \mapsto(2 n-1) t+1-i \\
& e_{1}^{1} \mapsto(2 n-1) t+1
\end{aligned}
$$

Dengan demikian, himpunan label titik pada subgraf $P_{n+1}^{i} \subseteq \operatorname{Amal}\left\{C_{n}\right\}_{t}$ untuk $i=1,2, \ldots, t$ adalah $g_{1}\left(V\left(P_{n+1}^{i}\right)\right) \cup g_{2}\left(E\left(P_{n+1}^{i}\right)\right)=N_{i}$ dan $g_{1}\left(V\left(P_{n+1}^{t}\right)\right) \cup$ 
$\overline{g_{2}\left(E\left(P_{n+1}^{t}\right)\right)=N_{t} \text {. Oleh karena itu, } \operatorname{Amal}\left\{C_{n}\right\}_{t} \text { memuat dekomposisi } P_{n+1} \text { super }}$ ajaib dengan konstanta ajaib $k=2 n t(n-1)+(3 n+t+1)$.

\section{KESIMPULAN DAN SARAN}

Amalgamasi titik $t$ buah graf siklus yang berukuran $n$ memuat dekomposisi super ajaib berbentuk lintasan dengan panjang $n+1$ mempunyai konstantan ajaib $k_{1}=2 n t(n-1)+(3 n+t+1)$ dan $k_{2}=2 n t(n-1)+(3 n+t+1)$.

Penelitian ini dapat dilanjutkan dengan menentukan dekomposisi ajaib untuk berbagai hasil operasi dari beberapa graf serta mencari dualnya.

\section{DAFTAR RUJUKAN}

Inayah, N., Lladó, A., \& Moragas, J. (2012). Magic and antimagic Hdecompositions. Discrete Mathematics, 312(7), 1367-1371. https://doi.org/10.1016/j.disc.2011.11.041

Maryati, T. K. (2011). Karakterisasi graf h ajaib dan graf h ajaib super. Institut Teknologi Bandung.

Maryati, T. K., Salman, A. N. M., \& Baskoro, E. T. (2013). Supermagic coverings of the disjoint union of graphs and amalgamations. Discrete Mathematics, 313(4), 397-405. https://doi.org/10.1016/j.disc.2012.11.005

Roswitha, M., Baskoro, E. T., Maryati, T. K., Kurdhi, N. A., \& Susanti, I. (2013). Further results on cycle-supermagic labeling. AKCE Int. J. Graphs Comb., 10(2), 211-220.

Salman, A. N. M., \& Maryati, T. (2010). On graph-(super) magic labeling of a path amalgamation of isomorphic graphs. In Proceeding of the 6th ICMSA 2010. Malaysia. 